\title{
Solving the Truck and Trailer Routing Problem with Fuzzy Constraints
}

\author{
Isis Torres ${ }^{1}$, Carlos Cruz $^{2}$, José L. Verdegay ${ }^{2}$ \\ ${ }^{1}$ Department of Software Engineering, \\ Instituto Superior Politécnico José Antonio Echeverría, 11500 Havana, Cuba \\ E-mail: itorres@ceis.cujae.edu.cu \\ ${ }^{2}$ Department of Computer Science and Artificial Intelligence, \\ University of Granada, 18071 Granada, Spain \\ E-mail: $\{$ carloscruz,verdegay\}@ decsai.ugr.es
}

Received 3 January 2015

Accepted 21 March 2015

\begin{abstract}
The Truck and Trailer Routing Problem consists of a heterogeneous fleet composed of trucks and trailers to serve a set of customers. This problem has been solved previously considering accurate data available. But in a real-world the available knowledge about some data and parameters involves a vague nature. In this study, we propose the application of the Soft Computing to model and solve the TTRP when capacity constraints are imprecise. Numerical examples are presented to illustrate the proposed method.
\end{abstract}

Keywords: Truck and Trailer Routing Problem, Fuzzy constraints, Soft Computing, Fuzzy Optimization

\section{Introduction}

Optimization of the movement of freight is vital to countries' future competitiveness in the global marketplace at this time. The Vehicle Routing Problem (VRP) is the most important mathematical problem in this field. The problem consists in designing the optimal set of routes for a fleet of vehicles in order to serve a given set of customers.

Truck and Trailer Routing Problem (TTRP) is an interesting extension of the VRP, defined by Chao ${ }^{7}$ in 2002, that adds the use of trailers to vehicle fleet. In this standard problem, customers are geographically dispersed and they have an associated demand. Customers are serviced by a fleet of vehicles (truck pulling a trailer) with known capacity. A truck plus a trailer is called a complete vehicle, and a vehicle without a trailer is called a pure truck. However, due to practical constraints, including government regulations, limited manoeuvering space at customer site, road conditions, etc., some customers may only be serviced by the truck. Then, TTRP considers two different kinds of customers: a customer who is accessible with or without a trailer is called a vehicle customer (VC) and one who is only accessible without a trailer is called a truck customer (TC).

Three types of routes can be defined in a TTRP solution as illustrated in Figure 1. Routes originating from and terminating at a central depot. Each type of route is limited by the capacity of vehicle used. The types of routes are:

- Complete Vehicle Route (CVR): Consisting of a main tour travelled by a complete vehicle, and at least one sub-tour travelled by the truck alone.

\footnotetext{
*Department of Software Engineering, Instituto Superior Politécnico José Antonio Echeverría, 11500 Havana, Cuba.
} 
- Pure Truck Route (PTR): This type of route is travelled by a truck alone and both customer types are visited.

- Pure Vehicle Route (PVR): It is the tour travelled by a complete vehicle and contains only vehicle customer without any sub-tour.

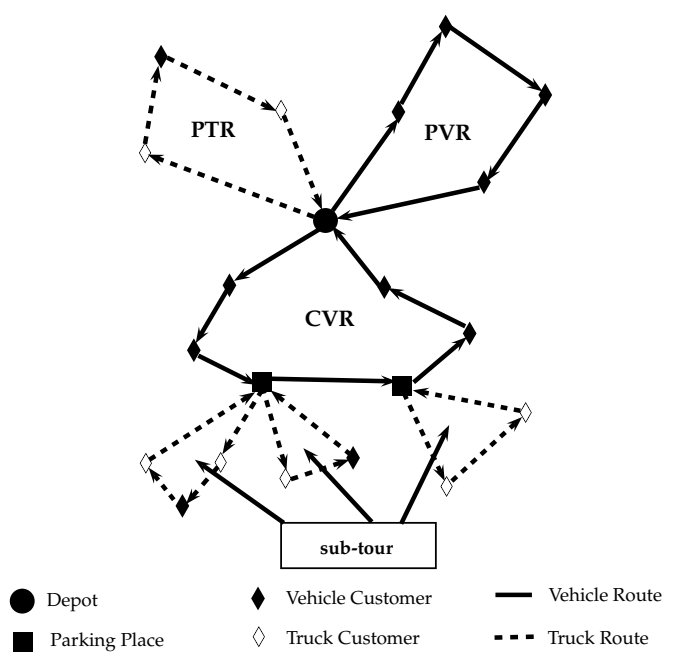

Fig. 1. Routes types in the TTRP

Since we have to consider different vehicle types in the TTRP, it makes sense to classify a route according to the vehicle serving it. A route is defined as a vehicle route $\left(R_{c v}\right)$ if the assigned vehicle is a complete vehicle, otherwise the route is referred to as a truck route $\left(R_{p t}\right)$ since it is serviced by a truck.

Besides this standard definition, there are a broad range of many TTRP variants and extensions, such as: Truck and Trailer Routing Problem with Time Windows (TTRPTW) ${ }^{20}$, Relaxed Truck and Trailer Routing Problem (RTTRP) ${ }^{19}$ and Single Truck and Trailer Routing Problem with Satellite Depots (STTRPSD) ${ }^{31}$. Also, there are others, such as Generalized Truck and Trailer Routing Problem (GTTRP) ${ }^{12}$ and Extended Truck and Trailer Routing Problem (ETTRP) ${ }^{34}$.

At this time, there is a growing interest in this problem motivated by its practical relevance to many real world problems such as milk collection ${ }^{6},{ }^{16},{ }^{17}$, ${ }^{23}$, food distribution ${ }^{14},{ }^{26}$ or postal delivery ${ }^{1}$ among other problems.

But in the real world these problems are complex and the information is not always available with suf- ficient precision and completeness as desired for adequate planning and management. It would be most noticeable in several emerging countries where the transport infrastructure is lacking: bad roads, neglected maintenances, poor technical state of trucks, low availability of resources, etc. In these circumstances the expressions are "about 50 units" or "no more than 65 units" etc. are usual respond to customer demand, And the same can happen with the rest of the parameters that define the problem such as travel times ("around 2-2:30 hours"), vehicle capacity ("no less than $3000 \mathrm{kgs",} \mathrm{"about} 100 \mathrm{kgs}$ extra load"). etc. The application of fuzzy logic is a recognized way to describe mathematically all these situations.

With this in mind, the goal of this paper is to solve the TTRP when the decision-maker is willing to permit some violations in the accomplishment of capacity constraints. For this, a model of TTRP with fuzzy constraints is proposed. Despite its importance, unfortunately until now not much research has been conducted in this problem using this point of view.

The method used to solve this model of the problem is a parametric approach ${ }^{30}$ used widely for fuzzy routing problems as well as other different fuzzy optimization problems. It is will be described in detail in next section. In order to corroborate the proposal, an extensive computational experiment has been carried out with 21 instances of the problem taken from ${ }^{7}$.

Thus, the paper is organized as follows. A review of the relevant literature about this problem is described in Section 2, while Section 3 is devoted to present one formal definition of TTRP. Section 4 proposes the fuzzy model for TTRP and describes a parametric approach for solving it. Results of the computational experiments on several problem instances are discussed in Section 5. Finally, conclusions are presented in Section 6.

\section{Literature review}

There are some previous works that modelled problems with characteristics similar to the TTRP but using other terms and denominations of the prob- 
lem. Thus, an approach was presented by Semet and Taillard in $1993^{26}$ to solve a problem of one of the major chain stores in Switzerland. The company needed to supply to a set of dispersed stores in the territory. It had a fleet of trucks and trailers and most of the stores were located in areas that did not admit access with trailers. The goal was to determine a transportation schedule using a heterogeneous fleet that minimizes the transportation costs. A Partial Accessibility Constrained Vehicle Routing Problem (PACVRP) ${ }^{25}$ was modeled in 1995. PACVRP is an extension of the VRP and takes into account the partial accessibility constraint introduced in the previous work ${ }^{26}$. In this case, the role of the partial accessibility constraint is to forbid the truck-customers. Another work is the study carried out by Gerdessen in 1996 on the Vehicle Routing Problems with Trailers (VRPT) ${ }^{14}$. In this problem the combination of truck and trailer was called vehicle or complete vehicle. However, the use of a complete vehicle could encounter manoeuvring problems at certain customer sites and therefore the trailer is parked. Time and trouble could be saved if these customers were served by the truck only. Also, an additional advantage would be the saving of fuel and driving faster. The VRPT is very similar to the current TTRP although the author simplifies it with a set of assumptions.

Finally, the term "Truck and Trailer Routing Problem" was first used in 2002 by Chao ${ }^{7}$ using a 2-phase approach to solve it. In the first phase a construction method was used to obtain an initial solution. This method assumes a relaxed generalized assignment, route construction, and descendent improvement. The initial solution is improved by a Tabu Search (TS) algorithm in the second phase. Similar to this work, Scheuerer in $2006^{24}$ applied a TS approach to address the same problem. Two new construction heuristics as multi-start procedures are developed in this case. The first constructive heuristic, called T-Cluster, is a clusterbased insertion heuristic that constructs routes sequentially. T-Cluster prioritizes the construction of complete routes vehicle. In the T-Sweep heuristic, feasible routes are constructed by rotating a ray centered at the depot and customers are gradually added to the current route, similar to the classical sweep algorithm by Gillett and Miller ${ }^{15}$.

Drexl in $2007{ }^{11}$ developed a branch and price algorithm to solve this problem. It is the only exact approach for this problem reported in the literature. The algorithm proposed was only tested on relatively small instances of the problem due to their NP-Hardness ${ }^{13}$.

In 2009, Lin et al. in ${ }^{18}$ developed a very effective Simulated Annealing (SA) based heuristic for the TTRP with three neighbourhoods. This proposal uses an indirect representation of the solution using a permutation the customers with additional artificial depot (dummy) to separate routes and terminate sub-tours. Also, it incorporates a vector of binary variables of length similar to the number of customers of complete vehicle to indicate the access way to these clients. The permutation is decoded with a procedure and the information of the binary vector into a TTRP solution. Since the decoding procedure could fail to find feasible solutions with respect to the availability of trucks and trailers then a procedure of combination of routes is applied to reduce the number of required trucks and trailers. Lastly, a term to penalize is added to the objective function to guide the search to feasible regions.

A mathematical programming based heuristic that also employs the cluster-first route-second approach ${ }^{5}$ was designed a year later. This method solves two sub-problems sequentially. The first called customer-route assignment problem (CAP) and the second, the route definition problem (RDP). The authors included these two models within an iterative mechanism that adds new constraints to the CAP based on the information of the RDP solution. This restarting mechanism is intended to diversify the search, and includes a TS mechanism that forbids (in the CAP) customers route assignments already explored in previous iterations of the algorithm.

Villegas et al. in 2011 solved the TTRP with a hybrid metaheuristic based on Greedy Randomized Adaptive Search Procedures (GRASP), Variable Neighborhood Search (VNS) and Path Relinking $(\mathrm{PR})^{32}$. The authors use a route-first, clustersecond (RFCS) procedure for the randomized con- 
struction of GRASP. The heuristic consists of three steps: (1) constructs a giant tour with a randomized nearest neighbour heuristic with a restricted candidate list of size $k,(2)$ an auxiliary acyclic graph $H=(X, U, W)$ is defined, and (3) solve the shortest path between the node 0 and $n$ of the graph $H$ for to obtain a TTRP solution. The procedure was adapted to take into account the accessibility constraint and the heterogeneous fixed fleet. The improvement phase of the hybrid metaheuristic is a VNS procedure that performs a classical variable neighbourhood descent step. The procedure VNS explores sequentially five neighbourhoods of a given TTRP solution using a best-improvement strategy. Within VNS infeasible solutions are accepted, provided that their infeasibility does not exceed a given threshold. Finally, hybridizing the PR with GRASP improves the performance of the latter. PR works as a mechanism to combine intensification and diversification.

Recently, a heuristic approach ${ }^{10}$ was applied to the TTRP which combined local search and large neighbourhood search as well as standard metaheuristics control strategies. Also, ${ }^{33}$ proposes a two phase metaheuristic that uses the routes of the local optima of a hybrid GRASP $\times$ ILS as columns in a set-partitioning formulation of the TTRP. Lastly, a revision of different methods used for this problem is presented in ${ }^{29}$.

\section{Formal definition of the problem}

The model discussed in this paper is based on the standard TTRP model proposed by I-M Chao. Following this definition, the TTRP can be formally defined on an undirected graph $G=(V, A)$, where $V=\{0,1,2, \ldots, n\}$, is a vertex set and $A=\left\{\left(v_{i}, v_{j}\right)\right.$ : $\left.v_{i}, v_{j} \in V, i \neq j\right\}$ is the set of edges. The vertices represent customer, except the vertex $v_{0}$ that corresponds to central depot. Each vertex of $V \backslash\{0\}$ has a non-negative demand $d_{i}$. The access constraints create a partition of $V=V_{c} \cup V_{t}$ into two subsets. $V_{c}=$ $\left\{v_{1}, v_{2}, \ldots, v_{l}\right\}$ is the subset of vertex representing the vehicle customers and $V_{t}=\left\{v_{l+1}, v_{l+2}, \ldots, v_{n}\right\}$ is the subset of vertex representing the truck customers.
$C$ is a matrix of non-negative cost. Each edge $(i, j)$ is associated with a cost $c_{i j}$ that represents the travel time required on the edge or the travel distance between vertex $i$ and vertex $j$. A heterogeneous fleet composed of $m_{c}$ trucks and $m_{r}$ trailers $\left(m_{c} \geqslant m_{r}\right)$ serves a set of customers. The capacities of the trucks and the trailers are $q_{c}$ and $q_{r}$ respectively. In general there are $m$ vehicles, of which $m_{r}$ are complete vehicles (truck pulling a trailer) and $m_{c}=m-m_{r}$ are trucks without trailers. A complete vehicle has a capacity $Q=q_{c}+q_{r}$. A route in the TTRP is composed of a partition of $V: R_{1}, R_{2}, \ldots, R_{m}$ and a permutation of $\delta_{m}$ specifying the order of the customers on route. Each route originating from and terminating at a central depot: $R_{m}=v_{0}, v_{1}, v_{2}, \ldots, v_{n+1}$, where $v_{0}=v_{n+1}$ denotes the depot. The cost in distance for a route performed with the vehicle $k$ is denoted as $C\left(R_{k}\right)$.

The goal of the TTRP is to minimize the total distance traveled by the fleet on all three types of routes and does not include any further cost components like fixed costs, costs for shifting demand between a truck and its pulling trailer, or costs for coupling or uncoupling of trailers. Also the routing costs do not differentiate between routes with and without trailers.

Lastly, a set of routes $R$ is a feasible solution for the TTRP if the routes start and end at the central depot and each customer is serviced exactly once. Also, the total demand of any vehicle route does not exceed the total capacity of the allocated vehicles used in that route; and the number of required trucks and trailers is not greater than available vehicles in the fleet

But, as we discussed above the case of modelling of vagueness in this problem has not been approached unfortunately until now.

\section{Fuzzy approach for the Truck and Trailer Routing Problem}

As said, TTRP is an optimization problem which by its nature favours the presence of vagueness, imprecision and uncertainty in the information handled. Nevertheless, the most of the models used for this problem in the literature assume that the data avail- 
able are accurate. For this reason, it would be appropriate to focus research toward defining TTRP models for incorporating the uncertainty present in the data.

In this paper, we deal with a TTRP where the decision maker tolerates violations in the accomplishment of the constraints; i.e. the decision maker permits the constraints to be satisfied "as well as possible". In these circumstances Soft Computing methodologies, and Fuzzy Logic in particular, has shown great potential in formulating and solving mathematical programming and decision making problems in the real world which are complex, ill-defined and not well understood.

Thus, following a work of Verdegay ${ }^{30}$, we use a general parametric approach to solve the TTRP with fuzzy constraints. This idea has been used widely for different fuzzy optimization problems. Some examples of the application to VRP are: ${ }^{2},{ }^{3},{ }^{4},{ }^{21}$. Also, extensions to the quadratic programming models appear in ${ }^{8},{ }^{27}$.

This parametric approach is composed of two phases: the first one transforms the fuzzy problem into several parametric problems in which a parameter $\alpha-$ cut represents the decision maker's satisfaction level. Then, in the second phase each of these $\alpha$-problems is solved by means of classical optimization techniques. The obtained results, to the different $\alpha$ values, generate a set of solutions and then all of these particular $\alpha$-solutions are integrated by the Representation Theorem for fuzzy sets. In this way the solution to a fuzzy problem has the same nature as the problem at hand, as stated by ${ }^{9},{ }^{22}$ and 30. The application of these phases for the TTRP with fuzzy constraints will be described hereinafter.

Each constraint of the problem can be represented as follows:

$$
a_{i} x \leqslant_{f} b_{i} \quad i \in M=\{1, \ldots, m\}
$$

where the symbol $\leqslant_{f}$ shall mean just that the decision-maker is willing to permit some violations in the accomplishment of the constraints up to a value of $b_{i}+\tau_{i}$ ( $\tau$ is referred to as a violation tolerance level). These violations are measured by means of membership functions: $\mu_{i}: \mathbb{R} \rightarrow[0,1]$ and these membership functions can be formulated as follows:

$$
\mu_{i}(x)= \begin{cases}1 & \text { if } a_{i} x \leqslant b_{i} \\ f_{i}\left(a_{i} x\right) & \text { if } b_{i} \leqslant a_{i} x \leqslant b_{i}+\tau_{i} \\ 0 & \text { if } b_{i}+\tau_{i} \leqslant a_{i} x\end{cases}
$$

Our proposal considers the constraints as fuzzy and admits the violation of these constraints according with a value of tolerance levels $\tau$, and $\alpha \in[0$, 1]. The fulfillment of the constraints can be verified using the following auxiliary model:

$$
a_{i} x \leqslant b_{i}+\tau_{i}(1-\alpha)
$$

Then the constraints associated to the capacity of the vehicles can be represented by:

$$
\begin{array}{ll}
\sum_{i=0}^{n} \sum_{j=i}^{n} d_{j} x_{i j}^{k 0} \leqslant_{f} Q & k=[1, \ldots, m] \\
\sum_{i=0}^{n} \sum_{j=1}^{n} d_{j} x_{i j}^{k 1} \leqslant{ }_{f} q_{c} \quad k & =[1, \ldots, m]
\end{array}
$$

where $x_{i j}^{k l}$ is a binary variable equal to 1 if and only if the vehicle $k$ with or without trailer $(l=0$ or $l=1)$ is used from $i$ to $j$, and 0 otherwise. Note that symbols $\leqslant_{f}$ denote that constraints (4) and (5) are considered as fuzzy and will be replaced by the following constraints:

$$
\begin{aligned}
\sum_{i=0}^{n} \sum_{j=i}^{n} d_{j} x_{i j}^{k 0} \leqslant Q+\tau_{1}\left(1-\alpha_{c c v r}\right) & k=[1, \ldots, m] \\
\sum_{i=0}^{n} \sum_{j=1}^{n} d_{j} x_{i j}^{k 1} \leqslant q_{c}+\tau_{2}\left(1-\alpha_{c c p r}\right) & k=[1, \ldots, m]
\end{aligned}
$$

where $\alpha_{c c v r}$ and $\alpha_{c c p r}$ are degrees of relaxation of each capacity constraint. In the constraint (7) the tolerance level $\tau_{2}$ incorporates the tolerance level of constraint (6), because this constraint verifies the truck and trailer capacity. Once the fuzzy problem is transformed into many classical problems, every problem can be solved using any of the strategies mentioned in Section 2. Results obtained for each $\alpha$ value generate a set of solutions and then the Representation Theorem for fuzzy sets can be used to integrate all these specific $\alpha$-solutions. 


\section{Computational experiments}

In this section, we present and discuss the computational experiments carried out to solve the TTRP with fuzzy constraints. It is important to emphasize that, at the present time, we are not trying to improve other solution methods for this kind of problems, but only to show the feasibility of our fuzzy approach. This is so because as far as we know there are no similar models in order to make a proper comparison of methods. All state of the art methods in the literature handle precise data.

As an illustrative example we have decided to use an approach based on the solution representation, the generating initial solution and the neighbourhood operators of ${ }^{18}$. Also, for the experiments we use 21 instances of testbed described by $\mathrm{Chao}^{7}$. Table 1 summarizes the characteristics of each problem used in these experiments.

The TTRP is a combinatorial optimization problem and is computationally more difficult to solve compared with the VRP. It cannot be solved in an exact way within a reasonable amount of time. Therefore, the main alternative to solving this class of problems is using approximate algorithms ${ }^{28}$. For this case we have implemented a Hill Climbing algorithm using Java and compiled using Eclipse 3.4.0.

We ran the experiments on a computer with an Intel Xeon running at $2.40 \mathrm{GHz}$ under Linux Ubuntu (version 3.2.0 on 64 bit x 86 SMP) with 23 GB of RAM. 30 independent runs were executed up to 100,000 fitness evaluations. The best solution found on each run was registered. The tolerance levels for each capacity constraint were calculated as $\tau_{1}=$ $10 \%$ of the capacity of the truck and $\tau_{2}=\tau_{1}+10 \%$ of the capacity of the trailer. Instances were solved for $\alpha \in\{0.0,0.1,0.2,0.3,0.4,0.5,0.6,0.7,0.8,0.9$, $1.0\}$.

Table 2 show the best value of the objective function obtained for each value of $\alpha-$ cut in 30 runs. Values contained in brackets are associated with the number of routes of each type (CVR, PVR, PTR) obtained in the solution. The last two rows of this table show the column average for all 21 problems and the number of times each $\alpha-$ cut found the best-known fuzzy solution (NBKFS). This column (NBKFS) for each $\alpha-$ cut computes the number of times got the best solution on the fuzzy variant of the 21 problems.

It can be seen that the average of minimum objective values from 30 runs of the $\alpha=0.0$ is slightly better than the remaining $\alpha$-cuts. Also, when $\alpha$ $=0.0$ it found 14 out of 21 NBKFS. Most the solutions are not feasible due to the fact that the number of vehicles used exceeds the number of available vehicles in the fleet. This is an aspect that would be interesting to tackle in future works, but is here beyond the objectives of this paper.

Note that there are cases $(1,5,7,9,14,17$ and 21) where the best solution found does not belong to the more relaxing problem $(\alpha=0.0)$. The relaxation of the problem (given by the value of $\alpha$-cuts or the tolerance value) sometimes does not imply a benefit. Basically the relaxation of the capacity constraints allows that for a given route more customers can visited than on the same route in the original problem. For instance, it can generate fewer routes where customers are more dispersed or equal number of routes but with unbalanced distribution of customers (final routes with few customers). Therefore, routes with many customers does not necessarily mean a lower cost. Also, in the instances 2 and 16 for $\alpha=1.0$ (crisp problem) the best solution is lower than in other $\alpha$-cuts. It is assumed that the problem with $\alpha=1.0$ should be the highest cost because there is no relaxation but as described above it does not always happen that way. This confirms that does not always relaxation of this problem involve a decrease in the cost of the solution. Moreover, for different $\alpha-c u t$ problems the solution obtained is equal in cost and number of routes. In this cases relaxation did not imply changes. In other words, relaxation of the problem did not involve a significant change in the solution cost. As can be seen in Figure 2 generally a further relaxation of the problem implies that the cost of the solution is less.

The averages obtained for each $\alpha-c u t$ in each problem are plotted in six graphs (see Figure 2). Each graph contains the behaviour average of the problems with equal numbers of customers. As can be seen the problems with a number of VC customers equal to TC customers $(2,8,11,14,17,20)$ 
Table 1: TTRP benchmark data reported by Chao 2002

\begin{tabular}{|c|c|c|c|c|c|c|c|}
\hline ID & Customers & $\mathrm{VC}$ & $\mathrm{TC}$ & Trucks & Capacity Truck & Trailers & Capacity Trailer \\
\hline 1 & & 38 & 12 & & & & \\
\hline 2 & 50 & 25 & 25 & 5 & 100 & 3 & 100 \\
\hline 3 & & 13 & 37 & & & & \\
\hline 4 & & 57 & 18 & & & & \\
\hline 5 & 75 & 38 & 37 & 9 & 100 & 5 & 100 \\
\hline 6 & & 19 & 56 & & & & \\
\hline 7 & & 75 & 25 & & & & \\
\hline 8 & 100 & 50 & 50 & 8 & 150 & 4 & 100 \\
\hline 9 & & 25 & 75 & & & & \\
\hline 10 & & 113 & 37 & & & & \\
\hline 11 & 150 & 75 & 75 & 12 & 150 & 6 & 100 \\
\hline 12 & & 38 & 112 & & & & \\
\hline 13 & & 150 & 49 & & & & \\
\hline 14 & 199 & 100 & 99 & 17 & 150 & 9 & 100 \\
\hline 15 & & 50 & 149 & & & & \\
\hline 16 & & 90 & 30 & & & & \\
\hline 17 & 120 & 60 & 60 & 7 & 150 & 4 & 100 \\
\hline 18 & & 30 & 90 & & & & \\
\hline 19 & & 75 & 25 & & & & \\
\hline 20 & 100 & 50 & 50 & 10 & 150 & 5 & 100 \\
\hline 21 & & 25 & 75 & & & & \\
\hline
\end{tabular}


Table 2: Results for 21 instances TTRP with fuzzy constraints

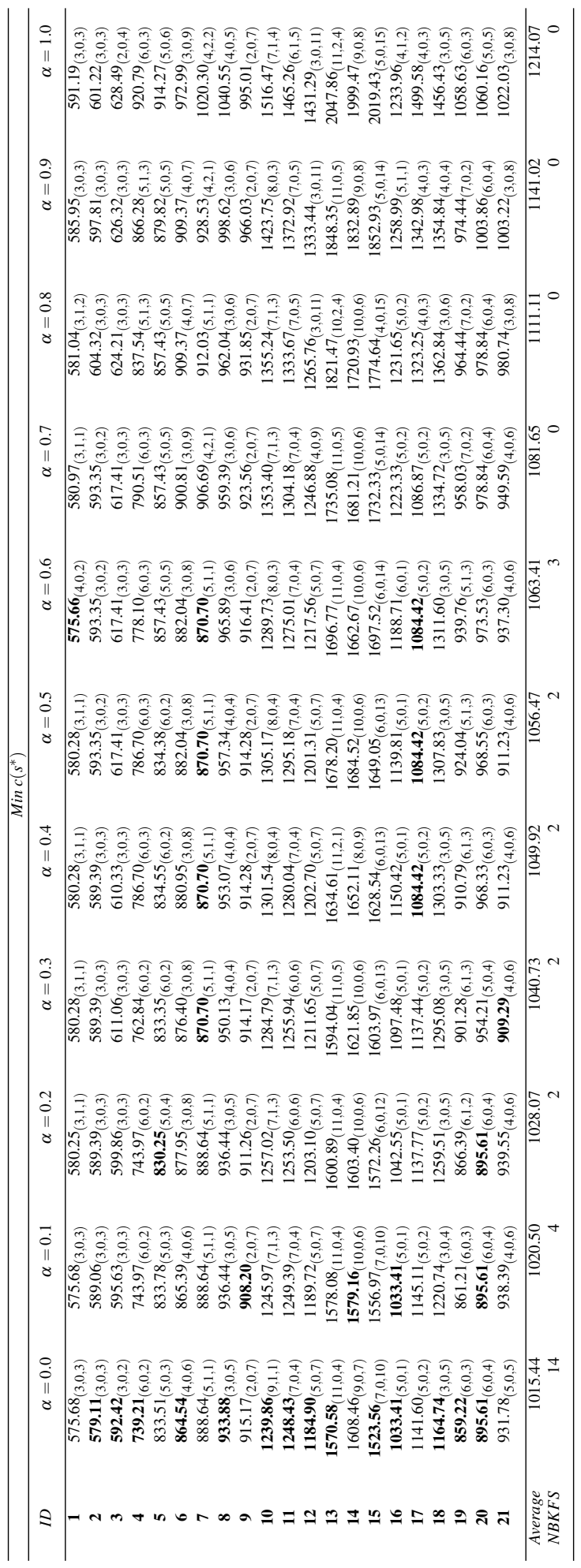


a) 50 customers

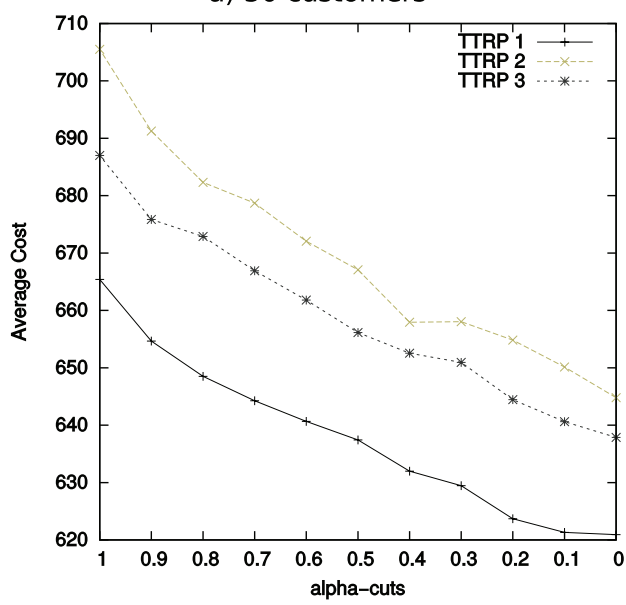

c) 100 customers

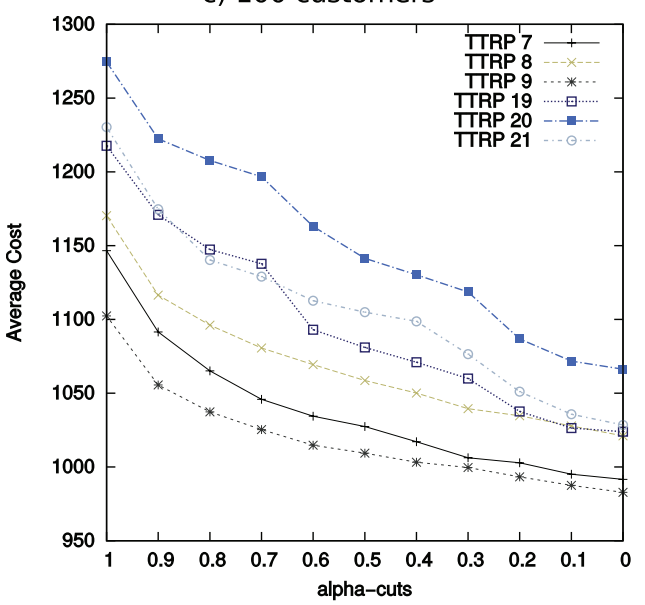

e) 150 customers

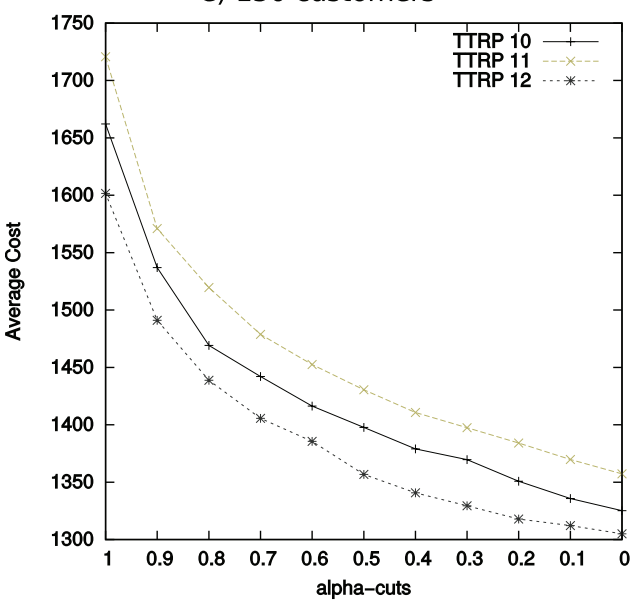

b) 75 customers

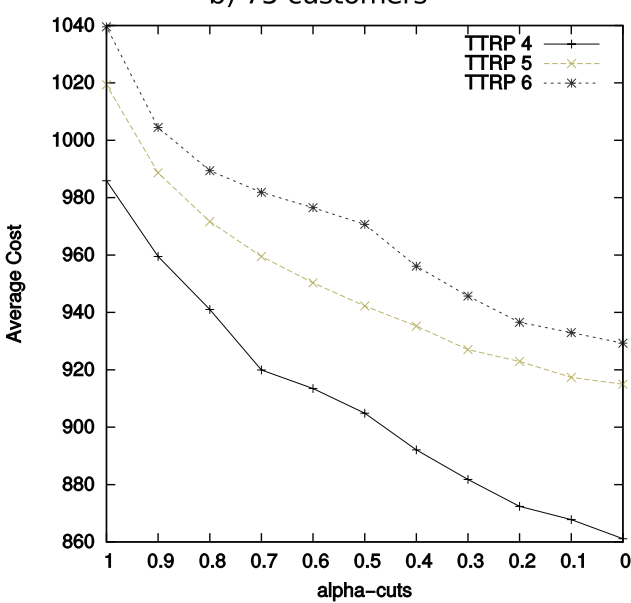

d) 120 customers
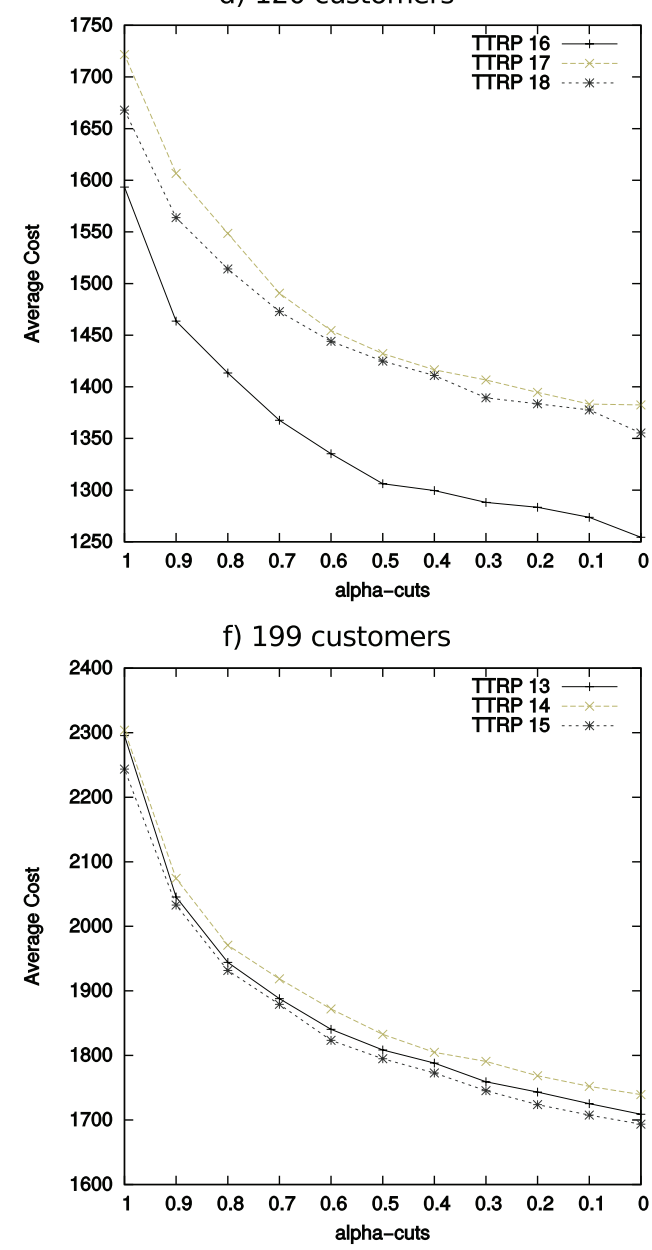

Figure 2: Behavior average of the solutions. 
Table 3: Average Rankings of the $\alpha$-cuts with Friedman test

\begin{tabular}{cc}
\hline \multicolumn{2}{c}{ Average } \\
\hline$\alpha-$ cuts & Ranking \\
\hline 1.0 & 11 \\
0.9 & 10 \\
0.8 & 9 \\
0.7 & 8 \\
0.6 & 7 \\
0.5 & 6 \\
0.4 & 4.9524 \\
0.3 & 4.0476 \\
0.2 & 3 \\
0.1 & 2 \\
$\mathbf{0 . 0}$ & $\mathbf{1}$ \\
\hline$p-$ value & 0
\end{tabular}

Table 4: Results of the Finner and Li test in the comparison between $\alpha$-cuts

\begin{tabular}{cccc}
\hline \multicolumn{5}{c}{ Average } \\
\hline$\alpha-$ cut & $p_{\text {Finner }}$ & $p_{L i}$ & Hypothesis \\
\hline 1 & 0 & 0 & Rejected \\
0.9 & 0 & 0 & Rejected \\
0.8 & 0 & 0 & Rejected \\
0.7 & 0 & 0 & Rejected \\
0.6 & 0 & 0 & Rejected \\
0.5 & 0.000002 & 0.000002 & Rejected \\
0.4 & 0.000161 & 0.000168 & Rejected \\
0.3 & 0.003631 & 0.004349 & Rejected \\
$\mathbf{0 . 2}$ & $\mathbf{0 . 0 5 6 1 7 1}$ & $\mathbf{0 . 0 7 0 2 0 7}$ & Not Rejected \\
$\mathbf{0 . 1}$ & $\mathbf{0 . 3 2 8 5 6 5}$ & $\mathbf{0 . 3 2 8 5 6 5}$ & Not Rejected \\
\hline
\end{tabular}


are the most expensive problems. This occurs in all cases except in graph 2(b).

Nonparametric tests for multiple comparison were used to better understand the results. These tests allow for checking if the results obtained by the $\alpha-$ cuts present any significant differences. Then, if there are differences, we use post-hoc procedures to compare the $\alpha-c u t$ of best ranking with the remaining $\alpha$-cuts. In this study we employ Friedman's test to analyze the average results of $\alpha-$ cuts. Table 3 shows the rankings of the different $\alpha-$ cuts that are considered in this study. The results indicate that the $\alpha=0.0$ has achieved the highest rankings.

Also, the results of the Friedman test revealed differences amongst the $\alpha-$ cuts in each problem. We have applied Finner's method and Li's method to compare the best ranking $\alpha=0.0$ with the remaining $\alpha$-cuts. Table 4 presents the results. The hypothesis of equality for the Friedman test has been rejected with a very small $p$-value. Therefore the results indicate that $\alpha=0.0$, with a significance level of 0.05 , dominates the other $\alpha-$ cuts in all cases except for $\alpha=0.1$ and $\alpha=0.2$.

Lastly, we perform a comparison between all $\alpha-$ cuts using the Friedman test for $N \times N$ comparison (always with significance of 0.05 ). The posthoc Nemenyi, Holm and Shaffer did not detect differences when the $\alpha$-cuts that were compared are very close. The results of of the test confirm that the level of relaxation in the problem can influence or not in the solution cost.

\section{Conclusion and future research}

Truck and Trailer Routing Problem is one of the most interesting problems that underlie the planning of logistics activities associated with transportation and distribution. In real-world activities, their implementation inevitably involves a degree of uncertainty or imprecision due to the lack of knowledge. In this case it makes perfect sense to think of fuzzy linear optimization based approaches for this problem. In contrast to what happens in the fuzzy formulation for Vehicle Routing Problems, unfortunately to date, not much research has been done in this important class of problems.
In this paper a parametric approach to deal with fuzzy constraints in the problem, and a heuristic was used to solve each classical problem obtained from the auxiliary model. Then, each $\alpha$-solution is integrated in a fuzzy solution. Thus, the decision maker would have a set of solutions according to their satisfaction degrees. The proposal was validated using a known benchmark for this kind of problems.

The authors aim to extend this research in future works in order to try to solve this problem when the coefficients of constraints are modeled as fuzzy numbers. Also, these models and methods will be used to solve real and practical business problems in future works.

\section{Acknowledgments}

This work was supported in part by the projects P11TIC-8001 from the Andalusian Government (including FEDER funds), TIN2011-27696-C02-01 from Spanish Ministry of Economy and Competitiveness, and to PYR 2014-9 GENIL Program from CEI BioTIC Granada, University fo Granada.

\section{References}

1. L. Bodin and L. Levy, "Scheduling of local delivery carrier routes for the United States postal service," Arc Routing: Theory, Solutions, and Applications, 419442 (2000).

2. J. Brito, F. J. Martínez, J. A. Moreno and J. L. Verdegay, "A GRASP-VNS Hybrid for the Fuzzy Vehicle Routing Problem with Time Windows," Proceedings of the Computer Aided Systems Theory - EUROCAST 2009, Lecture Notes in Computer Science, 5717, 825832 (2009).

3. J. Brito, F. J. Martínez, J. A. Moreno and J. L. Verdegay, "ACO-GRASP-VNS Metaheuristic for VRP with Fuzzy Windows Time Constraints," Proceedings of the $13^{\text {th }}$ International Conference on Computer Aided Systems Theory - EUROCAST 2011, Part I Lecture Notes in Computer Science, 6927, 440-447 (2012).

4. J. Brito, J. A. Moreno and J. L. Verdegay, "Transport route planning models based on fuzzy approach," Iranian Journal of Fuzzy Systems, 9(1), 141-158 (2012).

5. M. Caramia and F. Guerriero, "A heuristic approach for the truck and trailer routing problems," J. Oper. Res. Soc., 61(7), 1168-1180 (2010).

6. M. Caramia and F. Guerriero, "A Milk Collection Problem with Incompatibility Constraints," Inter- 
faces, 40(2), 130-143 (2010).

7. I-M. Chao, "A tabu search method for the truck and trailer routing problem," Comput. Oper. Res., 29(1), 33-51 (2002).

8. C. Cruz, R. C. Silva and J. L. Verdegay, "Extending and relating different approaches for solving fuzzy quadratic problems," Fuzzy Optim. Decis. Ma., 10(3), 193-210 (2011).

9. M. Delgado, J. L. Verdegay and M. A. Vila, "A general model for fuzzy linear programming," Fuzzy Set. Syst., 29(1), 21-29 (1989).

10. U. Derigs, P. Pullmann and U. Vogel, "Truck and trailer routing-problems, heuristics and computational experience," Comput. Oper. Res., 40(2), 536-546 (2013).

11. M. Drexl, "A branch and price algorithm for the truck and trailer routing problem," Technical Report, $N$. 27/2006 Deutsche Post Endowed Chair of Optimization of Distribution Networks, RWTH Aachen University, Germany, (2007).

12. M. Drexl, "Branch and price and heuristic colum generation for the generalized truck and trailer routing problem," Journal of Quantitative Methods for Economics and Business Administration, 12(1), 5-38 (2011).

13. M. R. Garey and D. S. Johnson, "Computers and Intractability: A guide to the theory of NPcompleteness," 338 pp. WH Freeman and Co., San Francisco (1979).

14. J. C. Gerdessen, "Vehicle routing problem with trailers," Eur. J. Oper. Res., 93(1), 135-147 (1996).

15. B. E. Gillett and L. R. Miller, "A heuristic algorithm for the vehicle-dispatch problem," Oper. Res., 22(2), 340-349 (1974).

16. A. Hoff and A. Lkketangen, "A tabu search approach for milk collection in Western Norway using trucks and trailers," Proceedings of the Sixth Triennial Symposium on Transportation Analysis, Phuket, Thailand (2007).

17. A. Hoff, "Milk collection in western Norway using trucks and trailers," Proceedings of the $5^{\text {th }}$ International Workshop on Freight Transportation and Logistics (ODYSSEUS 2012), Mykonos, Greece, 180-183 (2012).

18. S-W. Lin, V. F. Yu and S-Y. Chou, "Solving the truck and trailer routing problem based on a simulated annealing heuristic," Comput. Oper. Res., 36(5), 1638 1692 (2009).

19. S-W. Lin, V. F. Yu and S-Y. Chou, "A note on a the truck and trailer routing problem," Expert Syst. Appl., 37(1), 899-903 (2010).

20. S-W. Lin, V. F. Yu and C-C. Lu, “ A simulated annealing heuristic for the truck and trailer routing problem with time windows," Expert Syst. Appl., 38(12),
$15244-15252$ (2011).

21. B. Melian and J. L. Verdegay, "Using fuzzy numbers in network design optimization problems," IEEE T. Fuzzy Syst., 19(5), 797-806 (2011).

22. D. Ralescu, "Inexact solutions for large scale control problems," Proceedings of the First World Conference on Math at the Service of Man, Barcelona, Spain (1977).

23. S. Scheuerer, "Neue Tabusuche-Heuristiken für die logistische Tourenplanung bei restringierende Anhngereinsatz, mehreren Depots und Planungsperioden," Ph.D. Thesis, Wirtschaftswissenschaftliche Fakultät, Universität Regensburg (2004).

24. S. Scheuerer, "A tabu search heuristic for the truck and trailer routing problem," Comput. Oper. Res., 33(4), 894-909 (2006).

25. F. Semet, "A two-phase algorithm for the partial accessibility constrained vehicle routing problem," Ann. Oper. Res., 61(1), 45-65 (1995).

26. F. Semet and E. Taillard "Solving real-life vehicle routing problems efficiently using tabu search," Ann. Oper. Res., 41(4), 469-488 (1993).

27. R. C. Silva, C. Cruz and J. L. Verdegay, "Fuzzy costs in quadratic programming problems," Fuzzy Optim. Decis. Ma., 12(3), 231-248 (2013).

28. E-G. Talbi, "Metaheuristics: from design to implementation," 500 pp. John Wiley and Sons, New Jersey (2009)

29. I. Torres, J. L. Verdegay, C. Cruz and A. Rosete, "Models and solutions for truck and trailer routing problems: An overview," International Journal Applied Metaheuristic Computing, 4(2), 31-43 (2013).

30. J. L. Verdegay, "Fuzzy mathematical programming," In Fuzzy Information and Decision Processes, Eds. Gupta, M.M., Sanchez, E., 231-237 (1982).

31. J. G. Villegas, C. Prins, C. Cruz, C. Prodhon, A. L. Medaglia and N. Velasco, "GRASP/VND and multistart evolutionary local search for the single truck and trailer routing problem with satellite depots," Eng. Appl. Artif. Intel., 23(5), 780-794 (2010).

32. J. G. Villegas, C. Prins, C. Cruz, C. Prodhon, A. L. Medaglia and N. Velasco, "A GRASP with evolutionary path relinking for the truck and trailer routing problem," Comput. Oper. Res., 38(9), 1319-1334 (2011).

33. J. G. Villegas, C. Prins, C. Cruz, C. Prodhon, A. L. Medaglia and N. Velasco, "A matheuristic for the truck and trailer routing problem," Eur. J. Oper. Res., 230(2), 231-244 (2013).

34. R. Zitz, "Algorithms for the truck and trailer routing problem," Master's thesis, Department of Mathematics and Computer Science, University of Southern Denmark (2010). 